\title{
Akute respiratorische Infektionen: Neue Viren - weniger Therapie?
}

\author{
Acute Respiratory Infections: New Viruses, Less Therapy Options?
}

R. Urbanek

Institut

Zentrum für Kinder- und Jugendmedizin, Freiburg

\section{Bibliografie}

DOI $10.1055 / \mathrm{s}-0028-1083805$ Klin Pädiatr 2008; 220: 277-278

(c) Georg Thieme Verlag KG

Stuttgart · New York

ISSN 0300-8630

Korrespondenzadresse

Prof. Dr. R. Urbanek

Zentrum für Kinder- und

Jugendmedizin

Mathildenstr. 1

79106 Freiburg i. Br.

radvan.urbanek@

uniklinik-freiburg.de
Vor allem Säuglinge und Kleinkinder werden wegen Infekten der unteren Atemwege hospitalisiert. In den USA wird die Jahreshäufigkeit für Bronchiolitis auf 22,8/1000 Kinder jünger als 12 Monate geschätzt, wodurch erhebliche Kosten entstehen [6].

Die akuten respiratorischen Infektionen werden in ca. 90\% der Fälle durch Viren wie das Respiratory-Syncytial-Virus (RSV), Influenzavirus (IV) und Parainfluenzavirus (PIV), das humane Metapneumovirus (hMPV), Adenovirus, Rhinovirus, Enterovirus und, neu bekannt, auch durch das humane Bocavirus (hBoV) verursacht. Mittels Polymerase-Kettenreaktion (PCR) ist es heute möglich, den Erreger im nasopharyngealen Sekret schnell zu bestimmen [7]; gleichwohl soll aber bedacht werden, dass der Nachweis der viralen Nukleinsäure nicht über die Lebensfähigkeit des Erregers informiert.

Therapeutische Optionen sind - vor allem bei Bronchiolitis - kontrovers: Antivirale Medikamente, Bronchodilatatoren und Steroide. Bei einer Vielzahl der Erkrankten werden auch Antibiotika verabreicht, weil die Vorenthaltung der medikamentösen Therapie viel schwieriger ist, als diese Behandlung zu geben.

Fragen, die gestellt werden sind:

- Gibt es virusspezifische Symptome?

- Welche virale Infektion ist komplikationsträchtig?

- Ändert sich das Spektrum der viralen Erreger?

- Soll jedes Kleinkind mit Verdacht auf Infektion der unteren Atemwege geröntgt werden?

- Muss mit Folgeerscheinungen wie bronchiale Hyperreagibilität oder Asthma bronchiale gerechnet werden?

Um eine fundierte Antwort geben zu können, sind prospektive Studien erforderlich. Eines der Überwachungssysteme für akute respiratorische Infektionen (ARI) wird von Weigl et al. [9] dargestellt. Es zeigt sich eindeutig, dass aktive, zentral organisierte Erfassungssysteme den passiven überlegen sind. Die auf diese Weise im Netzwerk von drei großen Regionen erhobenen Daten waren sowohl repräsentativ als auch von prognostischer Signifikanz. In Deutschland verbreiten sich saisonale RSV- oder Influenzaepidemien schnell innerhalb von zwei Wochen. Die Kenntnis der Erreger hat daher eine besondere Bedeutung, da mit Palivizumab- oder Neuraminidaseinhibitoren vorgebeugt und behandelt werden kann. In der von Wasem et al. [8] in diesem Heft publizierten Studie wird einerseits über die Beständigkeit der viralen Erreger und andererseits über die Umsetzung der aktuellen diagnostischen und therapeutischen Leitlinien [2] berichtet. In einem konstanten Einzugsgebiet wurden über 1000 Kinder im Alter von 0-36 Monaten, die wegen akuter respiratorischer Infektion hospitalisiert wurden, über 6 Jahre prospektiv auf RSV, PIV 1, 2, 3 und IV A und B untersucht. Ein Drittel der nasopharyngealen Abstriche war für RSV, 8\% für PIV und $5 \%$ für IV positiv. Die epidemiologischen Daten der Jahre 1999-2001 haben sich nicht von denen der Jahre 2002-2005 unterschieden. Allerdings hat sich auch das angewandte therapeutische Vorgehen - trotz neuer Leitlinien - nicht wesentlich geändert. Lediglich die Häufigkeit der Röntgen-Thorax-Untersuchungen hat in der späteren Untersuchungsperiode nachgelassen.

Vor wenigen Jahren wurde über die Bedeutung des hMPV für die ARI im Kleinkindalter berichtet $[10,11]$, seit Kurzem wird auch die Wichtigkeit des weiteren viralen Erregers, des humanen Bocavirus, belegt. In der Publikation von Hengst et al. aus Aachen [4] wird über den aktuellen Virusnachweis in 173 Sekretproben von 162 Kindern informiert, die aufgrund schwerer, meist sauerstoffpflichtiger akuter respiratorischer Infektion stationär behandelt wurden. Ein Drittel der nasopharyngealen Aspirate war negativ, 54\% waren positiv für RS-, 9\% für Adeno-, hMP-, Iund PI-Virus sowie $10 \%$ für hBoV. Damit scheint dieser Virus eine bedeutsame Rolle bei akuten respiratorischen Infektionen zu spielen. Unklar ist noch, inwieweit die Pathogenität primär oder vor allem in Koinfektion mit RSV-Viren zum Tragen kommt [3].

Aus Schweden wurde berichtet [1], dass bei Kleinkindern mit hoher Viruslast an hBoV akute respiratorische Infektionen symptomatisch waren und bei denen mit niedriger Viruslast asymptomatische Verläufe beobachtet wurden. Auch eine serologische Untersuchung auf das humane Bocavirus, das in die Familie der Parvoviren gehört, wurde entwickelt [5]. Noch keine ausreichenden Kenntnisse bestehen über mögliche Folgeerscheinungen wie eine postinfektiöse Neigung zum Asthma.

Auf alle Fälle haben wir bei akuten respiratorischen Infektionen des Kleinkindes neue virale Erreger. Auch die Behandlung ändert sich. Gegenwärtige Leitlinien zur Bronchiolitistherapie haben bei Anwendung von Steroiden weder eine verkürzte Therapiedauer noch eine seltenere Notwendigkeit für Nachuntersuchungen belegt. Die Gabe von Steroiden wurde daher als nicht empfehlenswert eingestuft. Jetzt müssen wir nur entsprechend handeln, auch wenn uns dies bei den angestrengt atmenden Kindern und den besorgten Eltern sehr schwer fällt. 


\section{Literatur}

1 Allander T, Jartti T, Gupta S et al. Human Bocavirus and acute wheezing in children. Clin Infect Dis 2007; 44 (7): 904-910

2 AWMF-Leitlinien. Obstruktive Bronchitis in den ersten 3 Lebensjahren (incl. Bronchiolitis) AWMF-Leitinien Register-Nr. 026/00

3 Canducci F, Debiaggi M, Sampaolo M et al. Two-year prospective study of single infections and co-infections by respiratory syncytial virus and viruses identified recently in infants with acute respiratory disease. J Med Virol 2008; 80 (4): 712-723

4 Hengst $M$ et al. Infektionen mit dem humanen bocavirus (HBoV). Klin Pädiatr 2008; 220: 296-301

5 Kantola K, Hedman L, Allander T et al. Serodiagnosis of human Bocavirus infection. Clin Infect Dis 2008; 46 (4): 547-549

6 Leader S, Kohlhase K. Recent trends in severe respiratory syncytial virus (RSV) among US infants, 1997-2000. J Pediatr 2003; 143 (Suppl): S127-S132
7 Schauer U, Ilhorst G, Rohwedder A et al. Evaluation of respiratory syncytial virus detection by rapid antigen tests in childhood. Klin Pädiatr 2007; 219: 212-216

8 Wasem S, Weichert S, Walther $S$ et al. Lower respiratory tract disease in children: constant pathogens - constant management?! Klin Pädiatr 2008; 220: 291-295

9 Weigl JAI, Puppe W, Meyer CU et al. PID-ARI-net - A Pediatric Infectious Diseases Network on Acute Respiratory Infections and the added value of a multilevel research network. Klin Pädiatr 2008; 220: 281-286

10 Wilkesmann A, Schildgen O, Eis-Hübinger AM et al. Das humane Metapneumovirus als Erreger von Atemwegsinfektionen bei hospitalisierten Kindern - eine Übersicht. Klin Pädiatr 2007; 219: 58-65

11 Williams JV, Harris PA, Tollefson SJ et al. Human metapneumovirus and lower respiratory tract disease in otherwise healthy infants and children. N Engl J Med 2004; 350 (5): 443-450 